\title{
Identification of Motile Sperm Domain Containing 1 (MOSPD1) As a Novel Target of the Wnt/B-Catenin Signaling Pathway in Colorectal Cancer
}

Chiaki Horie

The University of Tokyo

Chi Zhu

The University of Tokyo

Kiyoshi Yamaguchi ( $\square$ kiyamagu@g.ecc.u-tokyo.ac.jp )

The University of Tokyo

\section{Saya Nakagawa}

The University of Tokyo

Kiyoko Takane

The University of Tokyo

\section{Tsuneo Ikenoue}

The University of Tokyo

\section{Yasunori Ohta}

The University of Tokyo

Yukihisa Tanaka

The University of Tokyo

\section{Susumu Aikou}

The University of Tokyo

Giichiro Tsurita

The University of Tokyo

\section{Yuka Ahiko}

The University of Tokyo

\section{Dai Shida}

The University of Tokyo

\section{Yoichi Furukawa}

The University of Tokyo

\section{Research Article}

Keywords: crucial role, MOSPD1, ChIP-qPCR, TCF-binding 
Posted Date: September 22nd, 2021

DOl: https://doi.org/10.21203/rs.3.rs-910485/v1

License: (c) (i) This work is licensed under a Creative Commons Attribution 4.0 International License. Read Full License 
1 Identification of motile sperm domain containing 1 (MOSPD1) as a novel

\section{target of the Wnt/ $\beta$-catenin signaling pathway in colorectal cancer}

3

4 Chiaki Horie ${ }^{1 \#}$, Chi Zhu ${ }^{1 \#}$, Kiyoshi Yamaguchi ${ }^{*}$, Saya Nakagawa1, Kiyoko

5 Takane ${ }^{1}$, Tsuneo Ikenoue ${ }^{1}$, Yasunori Ohta ${ }^{2}$, Yukihisa Tanaka², Susumu Aikou ${ }^{3}$,

6 Giichiro Tsurita ${ }^{3}$, Yuka Ahiko $^{3}$, Dai Shida ${ }^{3}$, Yoichi Furukawa $^{1}$

7

8 Division of Clinical Genome Research, Institute of Medical Science, The

9 University of Tokyo, Tokyo 108-8639, Japan

10 2Department of Pathology, Research Hospital, Institute of Medical Science, The

11 University of Tokyo, Tokyo 108-8639, Japan

12 '3epartment of Surgery, Research Hospital, Institute of Medical Science, The

13 University of Tokyo, Tokyo 108-8639, Japan

\#These authors contributed equally to this work.

*To whom correspondence should be addressed:

Kiyoshi Yamaguchi, Ph.D.

Division of Clinical Genome Research, Advanced Clinical Research Center Institute of Medical Science, The University of Tokyo

4-6-1 Shirokanedai, Minato-ku, Tokyo 108-8639, Japan

Phone: +81-3-6409-2106

Fax: +81-3-6409-2103 


\section{Abstract}

2 Aberrant activation of the Wnt/ $\beta$-catenin signaling pathway plays a crucial role in

3 the development and progression of colorectal cancer. Previously, we identified

4 a set of candidate genes that were regulated by this signaling pathway, and we

5 focused on MOSPD1, motile sperm domain containing 1 , in this study.

6 Immunohistochemical staining revealed that the expression of MOSPD1 was

7 elevated in tumorous cells of colorectal cancer tissues compared with non-

8 tumorous cells. Using ChIP-seq data and JASPAR database, we searched for

9 the regulatory region(s) in the MOSPD1 gene as a target of the Wnt/ 3 -catenin

10 signaling, and identified a region containing three putative TCF-binding motifs in

11 the 3'-flanking region. Additional analyses using reporter assay and ChIP-qPCR suggested that this region harbors an enhancer activity through an interaction with TCF7L2 and $\beta$-catenin. These data have clarified that MOSPD1 is a novel

14 direct target of the Wnt/ $\beta$-catenin signaling. In addition, the identification of its enhancer region may be helpful for the future studies of precise regulatory mechanisms of MOSPD1. 


\section{Introduction}

2 The Wnt/ $\beta$-catenin signaling pathway (also known as the canonical Wnt pathway)

3 is responsible for embryonic development and tissue homeostasis ${ }^{1}$. Aberrant

4 activation of this pathway by genetic and epigenetic alteration is involved in

5 human diseases such as cancer $^{2,3,4}$. In colorectal cancer (CRC), frequent

6 activation of $\mathrm{Wnt} / \mathrm{\beta}$-catenin signaling pathway by somatic mutations in APC

7 regulator of WNT signaling pathway $(A P C)$ or the $\beta$-catenin gene (CTNNB1) has

8 been reported. In the cBioPortal for Cancer Genomics

9 (https://www.cbioportal.org/), a public database of cancer genomes, mutations of

10 APC and CTNNB1 were found in $64 \%$ and $6 \%$, respectively, of 3,051 CRC tissues.

11 Loss of function mutations in APC or activating mutations in CTNNB1 results in

12 the stabilization and accumulation of $\beta$-catenin protein in the cells. The

13 accumulated $\beta$-catenin interacts with T cell factor (TCF) / lymphoid enhancer-

14 binding factor (LEF) transcription factors in the nucleus, and induces the

15 expression of their target genes (Wnt target genes) ${ }^{5}$. To date, more than one

16 hundred Wnt target genes have been identified, and a list of the genes is shown

17 on the Wnt homepage at https://web.stanford.edu/group/nusselab/cgi-

18 bin/wnt/target_genes. Studies of their function have helped to further understand 
1 the molecular mechanisms of carcinogenesis, and the complex regulatory

2 mechanisms underlying this signaling pathway. Representative examples of the

3 aberrant activation of this pathway contributing to carcinogenesis include MYC

4 proto-oncogene $(M Y C)$ and cyclin D1 (CCND1). MYC was identified by serial

5 analysis of gene expression using HT29 cells containing a zinc-inducible APC,

6 and affects a wide variety of functions such as cell proliferation, angiogenesis,

7 and promotion of anaerobic metabolism 6 . Cyclin D1 is known to regulate G1-S

8 cell cycle progression, and it was identified through the analysis of human genes

9 involved in controlling cell growth, the promoter regions of which contain the core

10 TCF/LEF-binding sites ${ }^{7}$.

It is of note that chromatin immunoprecipitation coupled with high-

12 throughput sequencing (ChIP-seq) analysis using six different cell lines and anti-

13 TCF7L2 antibody identified 116,000 non-redundant TCF7L2-binding sites, with

141,864 sites common to the cell lines tested, suggesting the existence of as yet

15 unidentified Wnt target genes in human cells ${ }^{8}$. To understand the precise

16 molecular mechanism underlying the development of Wnt-driven cancer, we

17 previously searched for new target genes by microarray using $\beta$-catenin-depleted

18 CRC cells and ChIP-seq of TCF7L2. Integrated analysis of these data identified 
111 candidate genes that are directly regulated by the $\beta$-catenin/TCF7L2 complex 9 .

2 Among these candidates, we focused in this study on motile sperm domain

3 containing 1 (MOSPD1), and revealed that MOSPD1 is a novel direct target of

4 the Wnt signaling pathway. Furthermore, we identified three Wnt responsive

5 elements in the 3'-flanking region of MOSPD1, and showed that the elements are

6 involved in the transcriptional activation. These data will help deepen our

7 understanding of colorectal carcinogenesis, as well as the regulatory mechanism

8 of MOSPD1.

9

11 Results

12 The expression of MOSPD1 is regulated by Wnt/ $\beta$-catenin signaling in

13 colorectal cancer cells

14 In the previous study, we identified a total of 11 target genes whose expression

15 was commonly down-regulated by the introduction of $\beta$-catenin siRNAs and a

16 dominant-negative form of TCF7L2 (dnTCF7L2) in HCT116, SW480, and LS174T

17 cells $^{9}$. Subsequent $\mathrm{qPCR}$ analysis revealed that the expression of PDE4D,

18 PHLDB2, OXR1, FRMD5, and MOSPD1 was significantly decreased by the 
1 knockdown of $\beta$-catenin. To verify the association of MOSPD1 with the $\mathrm{Wnt} / \beta$ -

2 catenin signaling, we performed western blot analysis using lysates from SW480

3 and HCT116 cells treated with $\beta$-catenin or control siRNA. In agreement with the

4 qPCR data, treatment with two independent $\beta$-catenin siRNAs decreased

5 MOSPD1 expression in both cells (Fig. 1a). In addition, treatment of HeLa cells

6 with lithium chloride ( $\mathrm{LiCl}$ ), a glycogen synthase kinase 3 (GSK3) inhibitor that

7 activates the $\mathrm{Wnt} / \beta$-catenin signaling, increased $\beta$-catenin and MOSPD1

8 expression (Fig. 1b). These data corroborated that MOSPD1 is a downstream

9 target of the $\mathrm{Wnt} / \mathrm{\beta}$-catenin signaling.

Since aberrant activation of the $\mathrm{Wnt} / \beta$-catenin signaling is involved in the

11 majority of $\mathrm{CRC}^{2,10}$, we searched for gene expression data of colorectal tumors

12 in NCBI Gene Expression Omnibus. In a dataset (GSE21510) containing 104

13 CRC tissues and 25 non-tumorous colonic tissues ${ }^{11}$, the average MOSPD1 expression was found to be 2.18-fold higher ( $q$-value: $3.05 \mathrm{E}^{-13}$ ) in the tumor tissues than in the non-tumorous tissues (Fig. 1c). In addition, the expression

16 levels showed a positive correlation with RNF43 $\left(r^{2}=0.40\right), A X I N 2\left(r^{2}=0.30\right)$, and MYC $\left(r^{2}=0.29\right)$, three well-known Wnt targets (Fig. 1d). These data supported that

18 MOSPD1 expression is induced by the activation of Wnt signaling. 
We further carried out immunohistochemical staining of $\beta$-catenin and

2 MOSPD1 using $11 \mathrm{CRC}$ tissues. As shown in Fig. 1e, $\beta$-catenin was stained in

3 the cytoplasm and/or nucleus of tumorous cells in all tumor tissues tested (upper

4 panel). In addition, MOSPD1 was also positively stained in the cytoplasm and/or

5 nucleus of the tumorous cells (Fig. 1e, lower panel).

\section{Identification of an enhancer in the 3'-flanking region of MOSPD1}

8 In our previous study, ChIP-seq analysis showed a region for the binding with

9 TCF7L2 in the 3'-flaking region of MOSPD1 (3'-putative enhancer, GRCh38-

10 chrX:134,885,306-134,886,672 $)^{9}$. This region was overlapped with a peak in

11 ENCODE ChIP-seq data of TCF7L2 (ENCSR000EUV, Fig. 2a, upper panel). In

12 addition to the 3'-region, the ENCODE data showed another peak in the 5'-

13 flanking region of MOSPD1 (GRCh38-chrX: 134,932,561-134,932,930). These

14 peaks were overlapped with peaks of histone modifications (H3K4me1:

15 ENCSR161MXP and H3K27Ac: ENCSR000EUT, Fig. 2a, middle and lower

16 panels), suggesting that these regions may have enhancer activity through the

17 interaction with TCF7L2. To investigate their enhancer activity, these regions

18 were cloned into reporter plasmids, and reporter assays were performed using 
1 HCT116 cells. As a result, both reporter plasmids, pGL4.23-MOSPD1-5'E and

2 pGL4.23-MOSPD1-3'E, showed increased reporter activity compared to the

3 mock reporter (empty) by 1.29- and 5.62-fold, respectively (Fig. 2b). Importantly,

4 co-transfection of the reporter plasmids with plasmids expressing a dominant-

5 negative form of TCF7L2 (dnTCF7L2) significantly decreased the reporter activity

6 of pGL4.23-MOSPD1-3'E, but not the activity of pGL4.23-MOSPD1-5'E,

7 suggesting the enhancer activity of the 3 '-flanking region through the interaction

8 with TCF7L2. In addition, knockdown of $\beta$-catenin by two independent siRNAs

9 markedly reduced the reporter activity of pGL4.23-MOSPD1-3'E (Fig. 2c). To confirm the interaction between the 3'-flanking region of MOSPD1 and

11 TCF7L2, we performed an additional ChIP-qPCR assay using anti-TCF7L2

12 antibody and region-specific primer sets for the 3'-enhancer region of MOSPD1.

13 An enhancer region in intron 2 of $R N F 43$ was recruited as a positive control ${ }^{12}$.

14 This assay detected an enrichment of the enhancer region in RNF43 by 4.53 -fold

15 in the precipitants with the anti-TCF7L2 antibody compared to those with normal

16 IgG. DNA fragments containing the 3'-enhancer region of MOSPD1 were 17 enriched by 10.3 -fold in the precipitants (Fig. 2d). These data suggested that both

18 TCF7L2 and $\beta$-catenin are involved in the enhancer activity and that TCF7L2 
1 associates with the 3'-enhancer region.

3 Involvement of three TCF-binding motifs in the enhancer activity

4 We further searched for TCF-binding elements (TBE) in the 3'-enhancer region

5 using JASPAR, a database for transcription factor binding profiles ${ }^{13}$, and

6 identified eight candidate TBEs (Supplementary Table S1). Among the eight, we

7 focused on three TBEs with a similarity score greater than 10; TBE1 (GRCh38-

$8 \quad$ chrX: 134,885,716-134,885,729), TBE2 (GRCh38-chrX: 134,885,543-

$9134,885,556)$, and TBE3 (GRCh38-chrX: 134,885,482-134,885,495). To

10 investigate the involvement of these motifs in the enhancer activity, we prepared

11 mutant reporter plasmids containing two-nucleotide substitutions in each TCF-

12 binding motif (TBE1-mut, TBE2-mut, and TBE3-mut) of pGL4.23-MOSPD1-3'E

13 and reporter plasmids containing these substitutions in the three motifs (TBEall-

14 mut) (Fig. 3a). A reporter assay determined that the reporter activity of mutant

15 plasmids (TBE1-mut, TBE2-mut, and TBE3-mut) was significantly reduced

16 compared to the wild type plasmids (pGL4.23-MOSPD1-3'E) by $9.87 \%, 35.3 \%$,

17 and $35.0 \%$, respectively. In addition, the activity of TBEall-mut plasmids was

18 markedly decreased compared to the wild type by $85.6 \%$ (Fig. 3b). Treatment of 
1 the cells expressing TBE1-mut, TBE2-mut, or TBE3-mut with $\beta$-catenin siRNA

2 suppressed the activity by $50.2 \%, 52.2 \%$, and $42.3 \%$, respectively, compared to

3 the cells with control siRNA. These data indicated that the three motifs are, at

4 least in part, associated with the enhancer activity of TCF7L2.

7 Discussion

8 In this study, we revealed for the first time that MOSPD1 is transcriptionally

9 regulated by Wnt signaling through the three TBEs located in its 3'-flanking region. MOSPD1 is a member of major sperm protein (MSP) domain-containing

11 family that is highly conserved in many species. There are three MSP domaincontaining proteins (MOSPD1, 2, and 3) in humans, and four (Mospd1, 2, 3, and 4) in mice and rats ${ }^{14}$. The similarities between human MOSPD1 and human

14 MOSPD2, and that between human MOSPD1 and human MOSPD3 are 8\% and $32 \%$, respectively, at protein levels (CLUSTALW, https://www.genome.jp/tools-

$16 \mathrm{bin} / \mathrm{clustalw})$. In our previous expression profile analysis, knockdown of $\beta$-catenin decreased MOSPD1 expression by $38 \%$ in SW480 cells, but it increased the expression of MOSPD2 and MOSPD3 by $20 \%$ and $40 \%$, respectively. These data 
1 may imply that MOSPD1 has a specific function that is linked with the canonical

2 Wnt signaling pathway in development. The function of MOSPD1 is still largely unclarified. In the early 1980s, MSP was isolated as a protein $15 \mathrm{~K}$ from sperm cells of Caenorhabditis elegans ${ }^{15}$,

5 implying its role in spermatogenesis. Later, MSP was shown to function as a

6 motility apparatus in sperm locomotion ${ }^{16,17}$. In GTEx Portal, a public database of

7 gene expression in normal tissues (https://gtexportal.org/home/), MOSPD1 is

8 expressed in a variety of tissues including esophageal mucosa, adrenal gland,

9 testis, skin, and uterus, suggesting that MOSPD1 should play physiological

10 role(s) in various tissues. In mice, Mospd1 is abundantly expressed in

11 mesenchymal tissues, and its expression is elevated during differentiation in

12 osteoblastic, myoblastic, and adipocytic cell lines ${ }^{14}$. Another study revealed that

13 Mospd1-null embryonic stem cells were able to proliferate and that they were

14 unable to differentiate to osteoblasts, adipocytes, and hematopoietic

15 progenitors ${ }^{18}$. These data indicated that Mospd1 should be involved in the

16 differentiation and proliferation of mesenchymal cells. In addition, knockdown of

17 Mospd1 induced the expression of epithelial cadherin Cdh1, and decreased the

18 expression of Snail1, Snai2, and mesenchymal cadherin Cdh11 in MC3T3-E1 
1 cells established from mouse osteoblasts ${ }^{14}$. These results suggested that

2 Mospd1 may be associated with epithelial-mesenchymal transition (EMT).

3 Interestingly, expression of Runx2 and Osteocalcin was also down-regulated by

4 the knockdown of Mospd1 in MC3T3-E1 cells ${ }^{14}$. RUNX2, one of the transcription

5 factors required for osteoblastic differentiation is abundantly expressed in the

6 nucleus of osteoid osteoma cells ${ }^{19}$. In addition, a study reported that surgical

7 resection of osteoid osteoma in two patients decreased total and

8 undercarboxylated osteocalcin in their sera, suggesting that osteocalcin is

9 secreted by the osteoid osteoma cells ${ }^{20}$. It is noteworthy that osteomas frequently

10 develop in the mandible bone of patients with germline variants in the APC gene ${ }^{21}$.

11 The induction of RUNX2 and/or osteocalcin by the increased expression of

12 MOSPD1 in osteoblasts may be involved in the development of osteomas in FAP

13 patients.

14 We have shown here that the expression of MOSPD1 is elevated in all

15 CRC tissues examined, and that its expression is associated with the

16 accumulation of $\beta$-catenin. The expression of MOSPD1 was shown to be

17 elevated in the circulating tumor cells of metastatic castration-resistant prostate

18 cancer $^{22}$. It was also reported that abnormal $\beta$-catenin expression was observed 
1 in approximately $38 \%$ of hormone-refractory prostate cancer, which is much

2 higher than that detected in prostate cancer tissues obtained from radical

3 prostatectomy ${ }^{23}$. Since MOSPD1 expression is associated with EMT, up-

4 regulation of MOSPD1 in cancer cells may be related to their invasion and

5 metastasis. In line with this view, ovarian cancer cells with high invasion-

6 phenotype expressed significantly increased levels of MOSPD1 compared to the

7 cells with low invasion-phenotype ${ }^{24}$. Although further studies are necessary,

8 augmented expression of MOSPD1 may play a crucial role in the EMT of CRC. We identified a distant enhancer region for the $\mathrm{Wnt} / \mathrm{\beta}$-catenin signaling in

10 the 3'-flanking region of MOSPD1. Enhancer regions that associate with $\beta$ -

11 catenin-TCF/LEF1 complexes have been identified in various regions of the

12 target genes. For instance, the enhancer regions of $M Y C^{6}, C C N D 1^{7}$, claudin-1

$13(C L D N 1)^{25}$, membrane-type matrix metalloproteinase $(M T 1-M M P)^{26}$, and $S P 5^{27}$

14 are localized in their 5'-flanking regions, and those of $R N F 43^{12}$ and $F R M D 5^{9}$ in

15 intron 2 and intron 1, respectively. Regarding $A X I N 2$, several enhancer regions

16 have been discovered in its $5^{\prime}$-flanking region and in intron $1^{28}$. It is of note that,

17 in addition to the 5'-flanking enhancer region, $M Y C$ has another enhancer

18 element in its $3^{\prime}$-flanking region ${ }^{29}$. Therefore, MOSPD1 may have additional 
1 enhancer region(s) in addition to the one identified here.

3 of the Wnt signaling pathway in CRC. Further analysis of MOSPD1 function will

4 elucidate the precise molecular mechanism underlying the development and

5 progression of $\mathrm{CRC}$, and may contribute to the development of therapeutic

6 strategies against their invasion and metastasis.

$9 \quad$ Materials and methods

10 Cell culture

11 Human CRC cell lines, HCT116 and SW480, and a human cervix cell line, HeLa were purchased from the American Type Culture Collection (Manassas, VA). All

13 cell lines were grown in appropriate media (McCoy's 5a Modified Medium for HCT116, Leibovitz's L-15 Medium for SW480, and Eagle's Minimum Essential Medium for HeLa) supplemented with $10 \%$ fetal bovine serum (BioSera,

16 Boussens, France), and antibiotic/antimycotic solution (Fujifilm Wako Pure

17 Chemical, Osaka, Japan). HCT116 and HeLa cells were maintained in 5\% $\mathrm{CO}_{2}$

18 at $37^{\circ} \mathrm{C}$, and $\mathrm{SW} 480$ cells were maintained without $\mathrm{CO}_{2}$ supplementation at $37^{\circ} \mathrm{C}$. 
$2 \quad$ Reporter plasmids and luciferase assay

3 Two genomic regions of 5'-putative (GRCh38-chrX: 134,932,384-134,933,013)

4 and 3'-putative enhancers (GRCh38-chrX: 134,885,255-134,886,704) were

5 amplified by PCR using region-specific primer sets and genomic DNA extracted

6 from the peripheral blood of healthy volunteers as a template. After digestion with

$7 \quad$ Xhol and Bg/ll restriction enzymes, the PCR products were cloned into pGL4.23

8 vector (Promega, Madison, WI) to generate pGL4.23-MOSPD1-5'E and

9 pGL4.23-MOSPD1-3'E. The primer sequences are shown in Supplementary

10 Table S2. HCT116 cells were transfected with these reporter plasmids together

11 with control or $\beta$-catenin siRNAs using Lipofectamine 2000 (Thermo Fisher

12 Scientific, Waltham, MA). pRL-null plasmids were co-transfected with the reporter

13 plasmids for normalization. 48 hours after the transfection, the cells were lysed

14 and reporter activities were measured using dual luciferase kit (TOYO B-Net,

15 Tokyo, Japan) and Lumat LB9507 Luminometer (Berthold Technologies, Bad

16 Wildbad, Germany). Firefly luciferase activities were normalized to Renilla

17 luciferase activity ( $p R L-n u l l)$. 


\section{$1 \quad$ Site-directed mutagenesis}

2 Mutant reporter plasmids containing substitutions in the consensus sequence of

3 the TCF7L2-binding motifs were prepared by site-directed mutagenesis. Wild

4 type-plasmid DNA of pGL4.23-MOSPD1-3'E was amplified using KOD-Plus-Neo

5 (Toyobo, Osaka, Japan) and a set of mutagenic primers (Supplementary Table

6 S3). The PCR products were digested with Dpnl restriction enzyme (Takara Bio,

7 Shiga, Japan) to cleave the methylated template DNA, followed by transformation

8 into Escherichia coli. Insertion of mutations in the plasmids was confirmed by

$9 \quad$ Sanger sequencing (3500xI DNA Analyzer, Thermo Fisher Scientific).

\section{Gene silencing}

12 For the knockdown of $\beta$-catenin, two $\beta$-catenin siRNAs (siß-catenin\#9: 5'GAUCCUAGCUAUCGUUCUU-3' and siß-catenin\#10: 5'-

14 UAAUGAGGACCUAUACUUA-3', Merck, Darmstadt, Germany) were used.

15 Control siRNA (siControl, ON-TARGET plus Non-targeting Pool, \#D-001810-10-

16 20) was purchased from Horizon Discovery (Lafayette, CO). Cells were 17 transfected with $10 \mathrm{nM}$ of the indicated siRNA using Lipofectamine RNAiMAX or 18 Lipofectamine 2000 (Thermo Fisher Scientific) for 48 hours. 


\section{Western blotting}

3 Total protein was extracted from cultured cells using SDS sample buffer (25 mM

4 Tris- $\mathrm{HCl}, \mathrm{pH} 6.8,0.8 \%$ sodium dodecyl sulfate, $4 \%$ glycerol). After boiling the

5 samples for $10 \mathrm{~min}$, the protein was separated by SDS-PAGE, and transferred

6 onto a nitrocellulose membrane (GE Healthcare, Buckinghamshire, UK). The

7 membranes were blocked with 5\% milk in TBS-T (Tris-buffered saline - Tween20),

8 and then incubated with primary antibody; anti-MOSPD1 (GTX32111, GeneTex,

9 Irvine, CA), anti- $\beta$-catenin (9582, Cell Signaling Technology, Danvers, MA), or

10 anti- $\beta$-actin antibodies (A5441, Merck). Horseradish peroxidase-conjugated goat

11 anti-mouse or anti-rabbit IgG (GE Healthcare) served as the secondary antibody

12 for the ECL Detection System (GE Healthcare).

\section{Chromatin immunoprecipitation assay}

15 Chromatin immunoprecipitation followed by qPCR (ChIP-qPCR) was performed

16 as described previously ${ }^{30}$. Briefly, HCT116 cells were cross-linked with $1 \%$

17 formaldehyde for $10 \mathrm{~min}$ at room temperature, and $0.1 \mathrm{M}$ glycine was added to

18 quench the formaldehyde. Chromatin was extracted and sheared by micrococcal 
1 nuclease digestion (New England Biolabs, Ipswich, MA). Subsequently, protein-

2 DNA complexes were immunoprecipitated with $10 \mu \mathrm{g}$ of anti-TCF7L2 antibody

3 (05-511, Merck) bound to Dynabeads Protein G (Thermo Fisher Scientific).

4 Normal mouse IgG (Santa Cruz Biotechnology, Santa Cruz, CA) was used as a

5 negative control. The precipitated protein-DNA complexes were purified by

6 conventional DNA extraction methods, and the purified DNA was subjected to

7 qPCR analysis using KAPA SYBR FAST ABI prism Kit (Kapa Biosystems,

8 Wilmington, MA) and a set of primers encompassing the TCF-binding motifs

9 located in the 3'-flanking region of MOSPD1. Amplification of a region upstream

10 of the GAPDH gene was used as a negative control. Sequences of the primers

11 are shown in Supplementary Table S4.

\section{Immunohistochemical staining}

14 Tissue sections were deparaffinized with xylene and rehydrated in a graded series of ethanol. Antigen retrieval was performed using $0.01 \mathrm{M}$ citrate buffer

$16(\mathrm{pH} 6.0)$ and autoclave heating at $110^{\circ} \mathrm{C}$ for $10 \mathrm{~min}$. After blocking endogenous peroxidase activity in $0.3 \% \mathrm{H}_{2} \mathrm{O}_{2}$ (Fujifilm Wako Pure Chemical) for 5 min, slides were incubated with $5 \%$ goat serum (ab7481, Abcam, Cambridge, UK) for 8 min, 
1 followed by the incubation with anti-MOSPD1 (GeneTex, 1:200) or anti- $\beta$-catenin

2 antibody (RB-1491, NeoMarkers, Union City, CA, 1:300) at $4^{\circ} \mathrm{C}$ overnight.

3 Secondary antibody, Dako EnVisionTM+ Dual Link System-HRP (Dako,

4 Carpentaria, CA), and ImmPACT DAB Substrate Kit (Vecter laboratories,

5 Burlingame, CA) were then used to visualize the immunoreactivity. Tissue

6 sections were counterstained with hematoxylin (Merck).

$7 \quad$ This study was approved by the ethical committee of the Institute of Medical

8 Science, The University of Tokyo (IMSUT-IRB, 2020-78-0318). All colorectal

9 tumor tissues and corresponding non-cancerous tissues were obtained with

10 informed consent from the resected specimens of patients who underwent

11 surgery or endoscopy. All methods were carried out in accordance with relevant

12 guidelines and regulations.

\section{Data Availability}

16 All data generated or analyzed during this study are included in this published 17 article. 


\section{Reference}

1. Clevers, H. Wnt/ß-catenin signaling in development and disease. Cell 127, 469-480 (2006).

2. Voloshanenko, O. et al. Wnt secretion is required to maintain high levels of Wnt activity in colon cancer cells. Nat. Commun. 4, 2610 (2013).

3. Wang, W. et al. Blocking Wnt secretion reduces growth of hepatocellular carcinoma cell lines mostly independent of $\beta$-catenin signaling. Neoplasia 18, 711-723 (2016).

4. Cleary, A. S., Leonard, T. L., Gestl, S. A. \& Gunther, E. J. Tumour cell heterogeneity maintained by cooperating subclones in Wnt-driven mammary cancers. Nature 508, 113-117 (2014).

5. Behrens, J. et al. Functional interaction of $\beta$-catenin with the transcription factor LEF-1. Nature 382, 638-642 (1996).

6. He, T. C. et al. Identification of c-MYC as a target of the APC pathway. Science 281, 1509-12 (1998).

7. Tetsu, O. \& McCormick, F. $\beta$-catenin regulates expression of cyclin D1 in colon carcinoma cells. Nature 398, 422-426 (1999).

8. Frietze, S. et al. Cell type-specific binding patterns reveal that TCF7L2 can be tethered to the genome by association with GATA3. Genome Biol. 13, R52 (2012).

9. Zhu, C. et al. Identification of FERM domain-containing protein 5 as a novel target of $\beta$-catenin/TCF7L2 complex. Cancer Sci. 108, 612-619 (2017).

10. Schatoff, E. M., Leach, B. I. \& Dow, L. E. Wnt signaling and colorectal cancer. Current Colorectal Cancer Reports 13, 101-110 (2017).

11. Tsukamoto, S. et al. Clinical significance of osteoprotegerin expression in human colorectal cancer. Diagnosis (2011). doi:10.1158/1078-0432.CCR10-2884

12. Takahashi, N., Yamaguchi, K., Ikenoue, T., Fujii, T. \& Furukawa, Y. Identification of two Wnt-responsive elements in the intron of RING finger protein 43 (RNF43) Gene. PLoS One 9, e86582 (2014).

13. Sandelin, A., Alkema, W., Engström, P., Wasserman, W. W. \& Lenhard, B. JASPAR: an open-access database for eukaryotic transcription factor binding profiles. Nucleic Acids Res. 32, (2004).

14. Thaler, R., Rumpler, M., Spitzer, S., Klaushofer, K. \& Varga, F. Mospd1, a new player in mesenchymal versus epidermal cell differentiation. J. Cell. Physiol. 226, 2505-2515 (2011). 
15. Klass, M. R. \& Hirsh, D. Sperm isolation and biochemical analysis of the major sperm protein from Caenorhabditis elegans. Dev. Biol. 84, 299-312 (1981).

16. Sepsenwol, S., Ris, H. \& Roberts, T. M. A unique cytoskeleton associated with crawling in the amoeboid sperm of the nematode, Ascaris suum. $J$. Cell Biol. 108, 55-66 (1989).

17. Nelson, G. A., Roberts, T. M. \& Ward, S. Caenorhabditis elegans spermatozoan locomotion: Amoeboid movement with almost no actin. J. Cell Biol. 92, 121-131 (1982).

18. Kara, M. et al. A Role for MOSPD1 in mesenchymal stem cell proliferation and differentiation. Stem Cells 33, 3077-3086 (2015).

19. Dancer, J. Y. et al. Expression of master regulatory genes controlling skeletal development in benign cartilage and bone forming tumors. Hum. Pathol. 41, 1788-1793 (2010).

20. Confavreux, C. B. et al. Osteoid osteoma is an osteocalcinoma affecting glucose metabolism. Osteoporos. Int. 2011235 23, 1645-1650 (2011).

21. Groen, E. J. et al. Extra-intestinal manifestations of familial adenomatous polyposis. doi:10.1245/s10434-008-9981-3

22. León-Mateos, L. et al. Global gene expression characterization of circulating tumor cells in metastasic castration-resistant prostate cancer Patients. J. Clin. Med 2020, 2066 (2066).

23. De La Taille, A. et al. $\beta$-catenin-related anomalies in apoptosis-resistant and hormone-refractory prostate cancer cells 1. (2003).

24. Puiffe, M.-L. et al. Characterization of ovarian cancer ascites on cell invasion, proliferation, spheroid formation, and gene expression in an in vitro model of epithelial ovarian cancer. Neoplasia 9, 820-9 (2007).

25. Miwa, N. et al. Involvement of claudin-1 in the $\beta$-catenin/Tcf signaling pathway and its frequent upregulation in human colorectal cancers. Oncol. Res. 12, 469-476 (2000).

26. Takahashi, M., Tsunoda, T., Seiki, M., Nakamura, Y. \& Furukawa, Y. Identification of membrane-type matrix metalloproteinase-1 as a target of the $\beta$-catenin/Tcf 4 complex in human colorectal cancers. Oncogene 21, 5861-5867 (2002).

27. Takahashi, M., Nakamura, Y., Obama, K. \& Furukawa, Y. Identification of SP5 as a downstream gene of the $\beta$-catenin/Tcf pathway and its enhanced expression in human colon cancer. Int. J. Oncol. 27, 1483-1487 (2005). 
1 28. Jho, E.-H. et al. Wnt/ß-catenin/Tcf signaling induces the transcription of Axin2, a negative regulator of the signaling pathway. Mol. Cell. Biol. 22, 1172-1183 (2002).

29. Yochum, G. S., Cleland, R. \& Goodman, R. H. A genome-wide screen for $\beta$-catenin binding sites identifies a downstream enhancer element that controls c-Myc gene expression . Mol. Cell. Biol. 28, 7368-7379 (2008).

30. Yamaguchi, K. et al. Overexpression of cohesion establishment factor DSCC1 through E2F in colorectal cancer. PLoS One 9, (2014). 


\section{Acknowledgements}

2 We thank Seira Hatakeyama and Yumiko Isobe (The University of Tokyo) for their

3 technical assistance. This work was supported in part by the Grant-in-Aid

$4 \quad$ \#20K07563 (K.Y.) from the Japan Society for the Promotion of Science.

6 Author information

$7 \quad$ Author contributions

8 The experiments were designed by K.Y., and Y.F. The experiments were

9 performed by C.H., C.Z., S.N., and Y.T. Data analysis was performed by C.H.,

10 C.Z., K.T., T.I., Y.O., S.A., G.T., Y.A., and D.S. The manuscript was written by C.H.,

11 K.Y., and Y.F. All authors critically read and approved the final paper.

\section{Additional information}

14 Conflict of interest

15 The authors declare no competing interests. 
$1 \quad$ Figure Legends

2 Figure 1. MOSPD1 is regulated by the Wnt/ $\beta$-catenin signaling in colorectal

3 cancer. (a) Suppressed expression of MOSPD1 by $\beta$-catenin siRNAs in SW480

4 and HCT116 cells. (b) Induction of $\beta$-catenin in HeLa cells treated with $\mathrm{LiCl}$ (30

$5 \mathrm{mM}$ and $100 \mathrm{mM}$ ) increased MOSPD1 expression. The expression of $\beta$-actin

6 served as a loading control. (c) Expression of MOSPD1 in 104 CRC tissues and

725 non-tumorous colonic tissues. The data were obtained from a dataset of

8 GSE21510 in GEO. Statistical significance was determined by unpaired t-test

9 with Benjamini-Hochberg correction. (d) Scatter plots show the positive

10 correlation between the expression levels of three Wnt target genes ( $Y$-axis) and

11 MOSPD1 (X-axis). The data were obtained from GSE21510. (e)

12 Immunohistochemical staining of $\beta$-catenin (upper) and MOSPD1 (lower) in CRC

13 tissues. Scale bars $100 \mu \mathrm{m}$. Full-length, uncropped images are included within

14 Supplementary Figure.

16 Figure 2. TCF7L2-interacting region in the 3'-flanking region may play a role

17 as an enhancer. (a) Schematic representation of the ENCODE ChIP-seq data of

18 TCF7L2, H3K4me1, and H3K27ac in HCT116 cells. (b) Reporter activity of 
1 putative enhancer regions in the 5'- and 3'-flanking regions. HCT116 cells were

2 transfected with pGL4.23 (Empty), pGL4.23-MOSPD1-5'E (MOSPD1-5'E), or

3 pGL4.23-MOSPD1-3'E (MOSPD1-3'E) plasmids, in combination with pRL-null

4 reporter plasmids for the normalization of transfection. The cells were co-

5 transfected with pCAGGS-dnTCF7L2 plasmids expressing a dominant-negative

6 form of TCF7L2 or the empty plasmids (Mock). Relative luciferase activities

7 represent mean \pm SD from three independent cultures. Statistical significance

8 was determined by Student's t-test or Dunnett's test. (c) Effect of $\beta$-catenin siRNA

9 on the reporter activity of pGL4.23-MOSPD1-3'E (MOSPD1-3'E) in HCT116 cells.

10 Relative luciferase activities represent mean \pm SD from three independent

11 cultures. Statistical significance was determined by Dunnett's test. (d) ChIP-

12 qPCR was performed using region-specific primer sets and the precipitants with

13 an anti-TCF7L2 antibody or those with normal IgG. The amplification of a region

14 upstream of GAPDH was used for normalization. The enhancer region in intron

152 of RNF43 was used as a positive control. Data represents mean \pm SD from

16 three independent experiments. A significant difference was determined by

17 Student's t-test. 
1 Figure 3. Involvement of the three TBEs in the reporter activity. (a)

2 Schematic representation of wild type (open circle: WWCAAAG, W: A/T) and

3 mutant (closed circle: WWCAGCG) TCF-binding motifs in pGL4.23-MOSPD1-3'E

4 reporter plasmids. (b) Relative reporter activity of empty, wild type and mutant

5 reporter plasmids in HCT116 cells (WT, TBE1-mut, TBE2-mut, TBE3-mut, or

6 TBEall-mut). The data represent mean \pm SD from three independent cultures.

$7 \quad$ Statistical significance was determined by Student's t-test or Dunnett's test. 


\section{Figure 1}

a

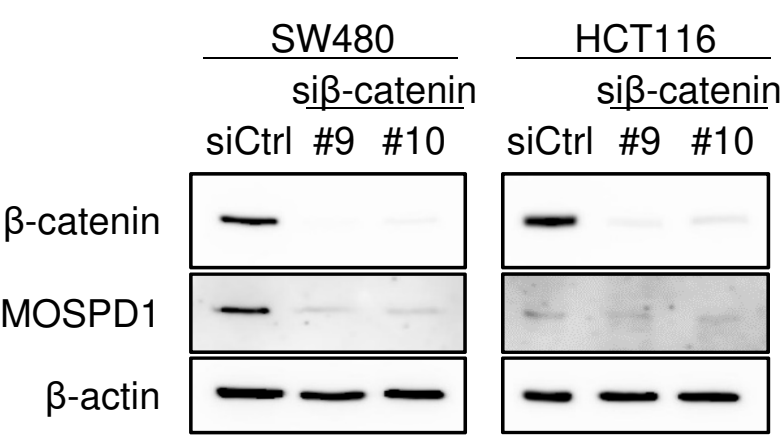

b

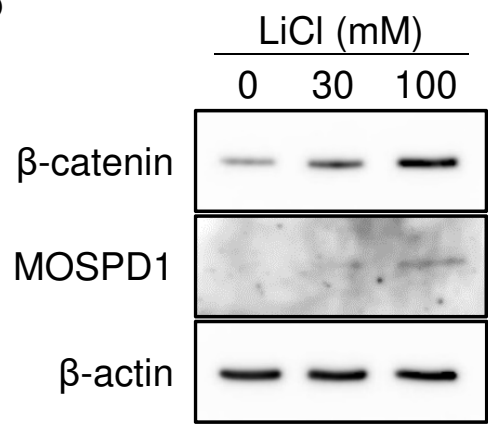

c
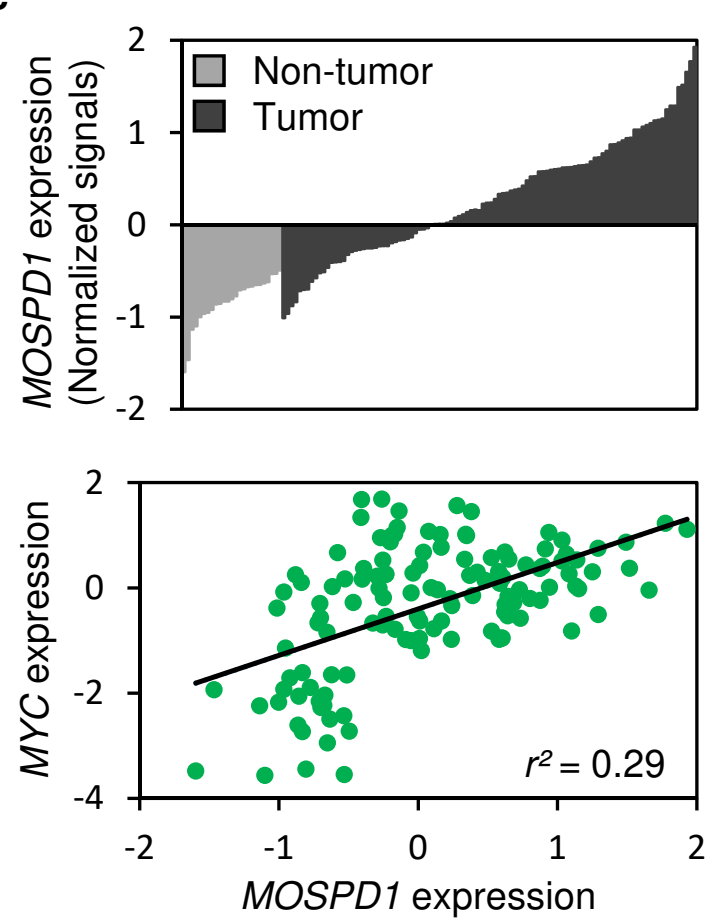

e
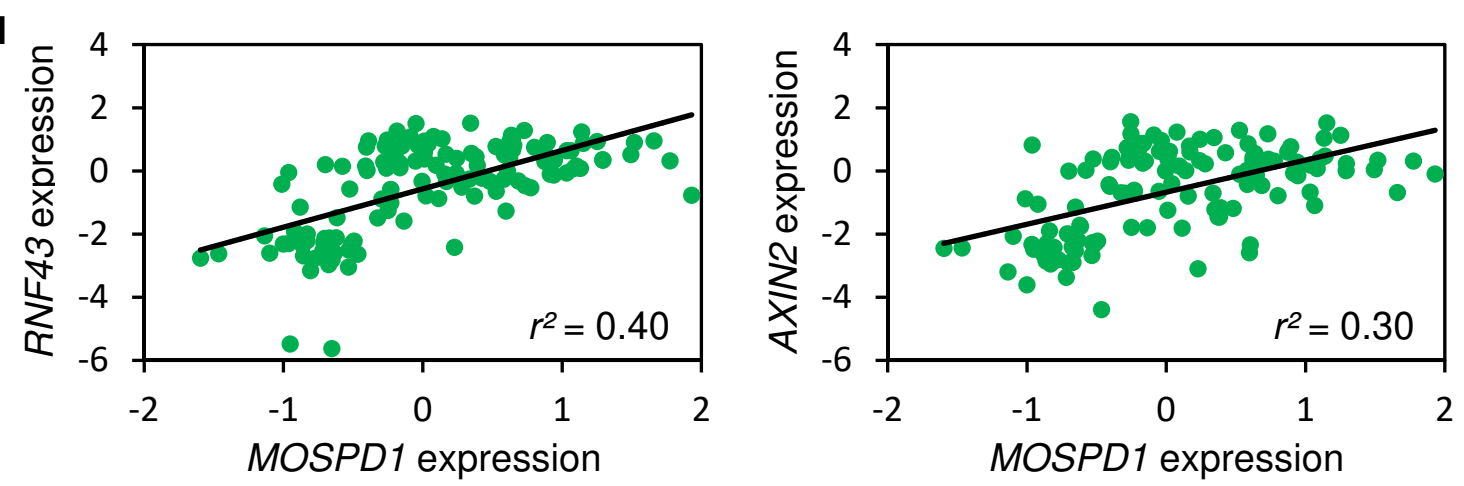

B12-00022

B21-00361

B32-00362

B21-00409

B21-00436

$\beta$-catenin

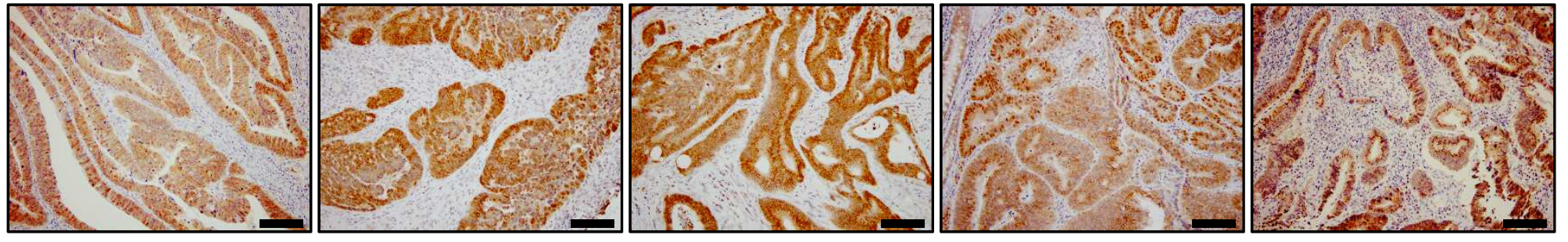

MOSPD1

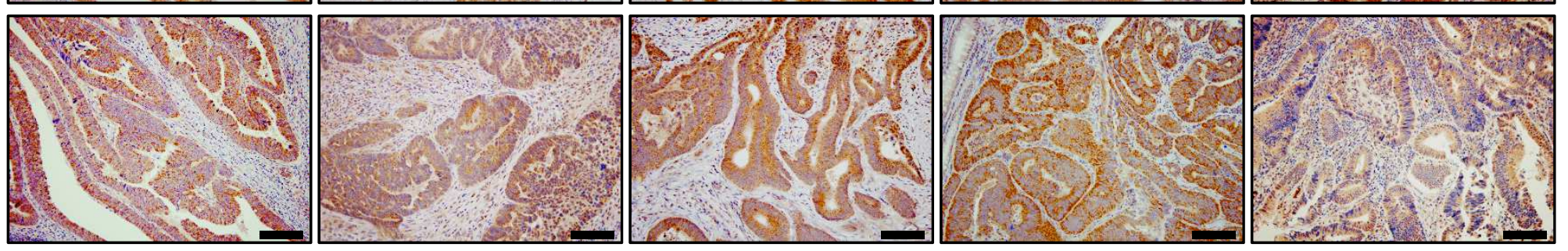


Figure 2

a

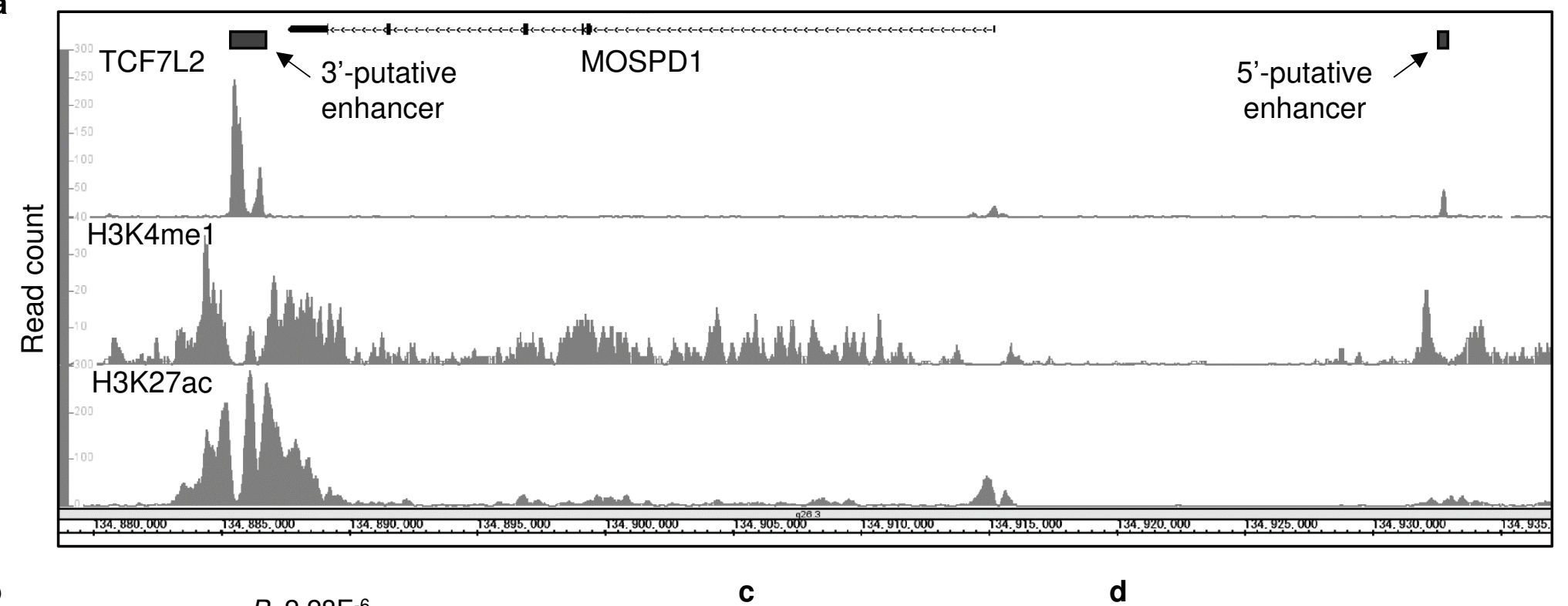

b

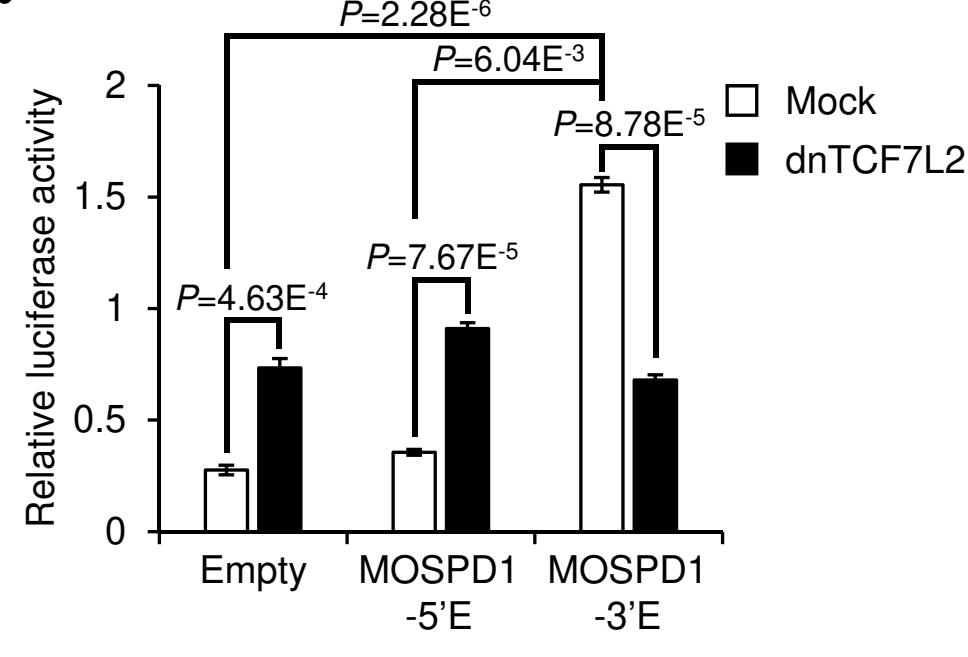

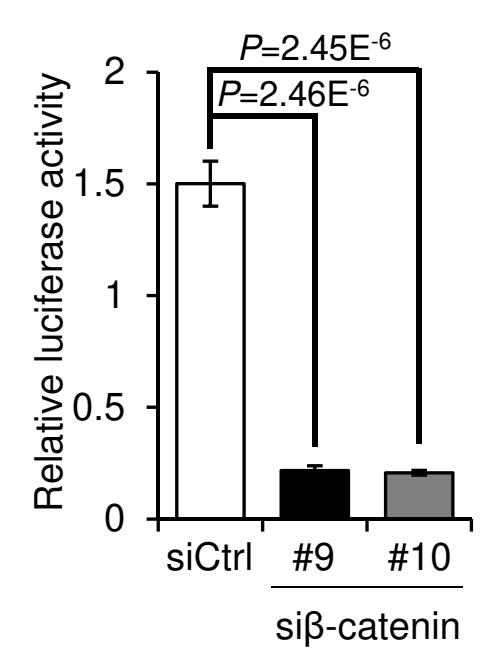

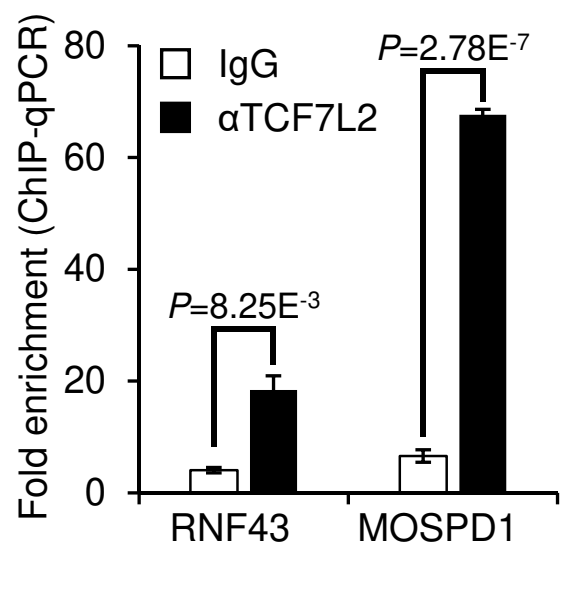


Figure 3

a

TBE1 TBE2 TBE3

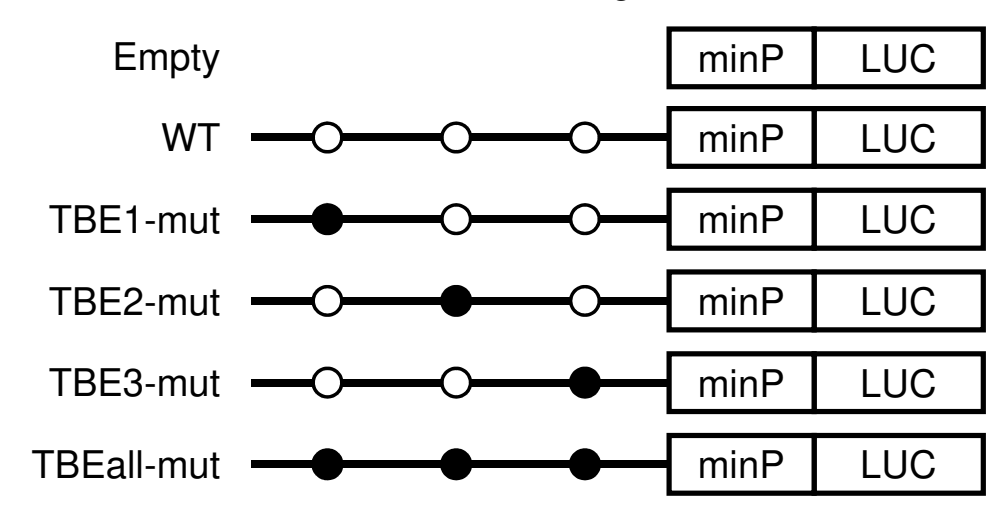

b

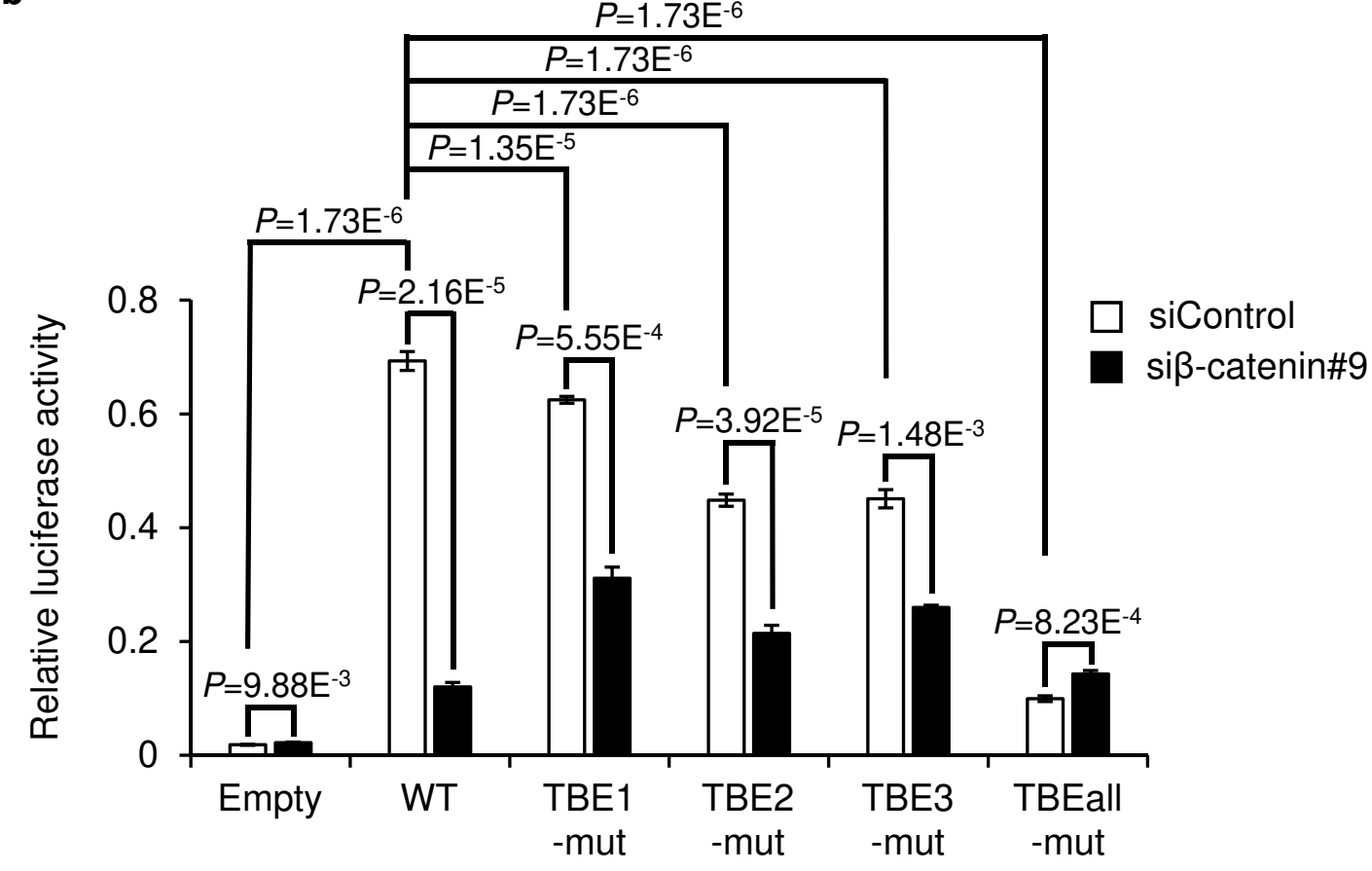




\section{Supplementary Files}

This is a list of supplementary files associated with this preprint. Click to download.

- Supplementarymaterials.pdf 